\title{
Skipped multilevel lesion as an atypical tuberculous spondylitis mimicking spinal metastasis: A case report
}

Tuberculosis (TB) is one of major global health threats and leads to morbidity and mortality. ${ }^{1} \mathrm{~TB}$ is prevalent in low and middle income countries especially in Sub-Saharan Africa and South East Asia. $^{2}$

M. tuberculosis usually affects the lungs. Spine is the most common extrapulmonary sites for TB after lymph nodes, accounts for $1 \%$ of all TB cases, and 50 to $60 \%$ of osteoarticular TB. ${ }^{2,3}$ In the case of spinal TB, two or more adjacent vertebrae are usually involved due to hematogenous spread of bacteria in which the vertebral arteries feed the two adjacent vertebrae. The incidence of multiplelevel non-contiguous spinal TB is 1.1 to $16 \%$ of all skeletal TB. ${ }^{2}$

Multiple-level non-contiguous, an atypical form of spinal TB is rare. It has insidious symptoms, often with diagnostic delays and a higher risk of permanent neurological deficits. ${ }^{4}$ We report a case of atypical TB spondylitis with non-contiguous multiple level of lesions mimicking multiple bone metastases.

\section{Case report}

A 20-year-old female presented with progressive weakness of her lower extremities lasting 3 months prior to admission and worsened in 2 weeks. Weakness was first felt at right lower extremity followed by left lower extremity. She experienced low back pain for 6 months and got worse at rest and numbness at the chest down to the leg for 1 month. There were urination and defecation disturbance for 1 week. On neurological examination, paraplegia of lower extremities was found. There were hypoesthesia below thoracic 8-9 spinal cord segment and proprioception disturbance on both lower extremities. Tendon reflexes were increased with extensor plantar responses on both feet. Laboratory results revealed normal complete blood count, but high erythrocyte sedimentation rate (ESR, $75 \mathrm{~mm} /$ hour) and high C-reactive protein (CRP, $111.19 \mathrm{mg} / \mathrm{L})$. Chest X-ray was within normal limits.

Spinal magnetic resonance imaging (MRI) showed spondylitis at the level of thoracic 2-4 and lumbar 2-4 vertebrae accompanied by intraosseus abscess formation. (Figure 1). The bone computed tomography (CT) and spinal MRI showed destruction of the corpus, pedicle and lamina at the level of thoracal 2-4 and lumbar 3 vertebrae (Figure 2). As tumor metastasis was a differential diagnosis, the patient underwent debridement treatment and posterior stabilization surgery. Histopatohology examination of the resected tissues revealed abundant caseous necrosis with granulomatous multinucleated giant cells consistent with tuberculous infection.

The patient was treated for 9 months with a combination of anti-TB medication, rifampicin (450 $\mathrm{mg}$ /day), isoniazid (300 mg/day), ethambutol (750mg/day) and pyrazinamide (750 mg/day). Motoric improvement was achieved three months following the treatment, she was able to walk without assistant.

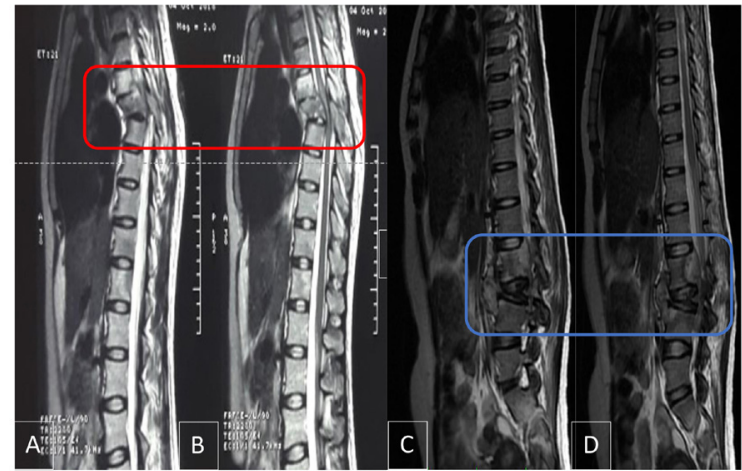

Figure 1. Spinal MRI 1.5 Tesla Sagittal of T2 showing Tuberculous Spondylitis Vertebrae $(A, B)$ Thoracal of 2-4 (C,D) Lumbar 2-4

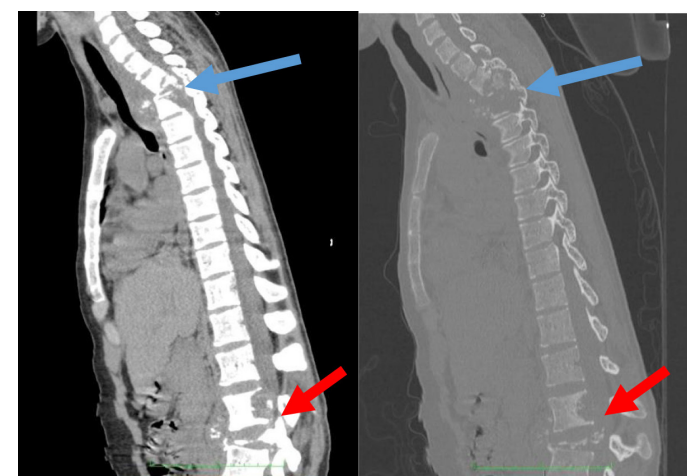

Figure 2. Bone Computed Tomography (CT) 
TB spondylitis is the most common form of the musculoskeletal TB. It is often associated with an insidious onset and non-specific initial symptoms. The local symptom is usually a back pain occurring long before a definitive diagnosis. Clinicians should be aware of the possibility of TB spondylitis if patients present with chronic back pain even without other systemic symptoms, in the endemic region..$^{4-6}$

TB can involve the spinal or other extrapulmonary sites due to spread of M. tuberculosis from the primary site of infection through hematogenous pathway including the arterial and venous systems. The vascular plexus plays a role in the spread of hematogenous infections in the paradiscal area. The Batson paravertebral venous plexus in the vertebra is a valveless system that allows blood to flow freely in both directions depending on the pressure generated by the intra-abdominal and intrathoracic cavities. The spread of infection through the intraosseous venous system may be responsible for vertebral central body lesions. ${ }^{7}$

The typical form of TB spondylitis can be easily diagnosed but there are recent increased incidence of atypical form. The increased diagnosis may be due to greater use of whole spine MRI to diagnose TB spondylitis. The incidence of non-contagious spinal TB also have a higher incidence in regions with a high prevalence of TB cases. ${ }^{8}$ CRP and ESR are important laboratory indicators for inflammation in spinal TB.$^{8-11}$ Bone or soft tissue biopsies may also be necessary to make a definitive diagnosis. ${ }^{8}$

TB spondylitis in adolescent is sometimes misdiagnosed as a neoplastic disease. Approximately $12 \%$ of patients with cancer present with spinal metastases, and the major sites of spinal lesions are the lower thoracic and upper lumbar regions similar to TB. ${ }^{8}$

The principle treatment of atypical spinal TB is similar to the typical cases. For the management of extrapulmonary TB of the bones and joints, duration treatment is extended to 9 months. Surgery is an efficient treatment for spinal TB patients with neurological deficits, kyphotic deformity or large abscesses. Patients with skipped non-contiguous lesions are more prone to develop neurological complications often requiring surgical intervention..$^{5,10,11}$

\section{Paulus Sugianto, Felisitas Farica Sutantoyo}

Department of Neurology, Faculty of Medicine Airlangga University, Dr. Soetomo General Hospital, Surabaya, Indonesia

Keywords: Tuberculous Spondylitis, atypical lesion, non-contiguous multiple lesion

Address correspondence to: Felisitas Farica Sutantoyo, MD, Department of Neurology, Airlangga University- Dr. Soetomo General Hospital, Surabaya 60286, East Java, Indonesia. Tel +6231-5501670-5501672, E-mail: felisitasfarica@ gmail.com

https://doi.org/10.54029/2021jun

\section{REFERENCES}

1. Ageyman A, Ofori R. Tuberculosis-An Overview. J Public Health Emerg. 2017;1(7):1-11

2. Ekinci S, Tatar O, Akpancar S, et al. Spinal tuberculosis. J Exp Neurosci. 2015;9:89-90.

3. Kaila R, Malhi AM, Mahmood B, et al. The incidence of multiple level noncontiguous vertebral tuberculosis detected using whole spine MRI. J Spinal Disord Tech. 2007;20:78-81.

4. Wang LN, Wang L, Liu LM, et al. Atypical spinal tuberculosis involved noncontiguous multiple segments: case series report with literature review. Medicine. 2017;96:e6559

5. Sivalingam J, Kumar A. Spinal Tuberculosis Resembling Neoplastic Lesion on MRI. JCDR. 2015;9(11):TC01TC03

6. Ye M, Huang J, Wang J, Ren J, Tu J, You W, et al. Multifocal musculoskeletal tuberculosis mimicking multiple bone metastases: a case report. BMC Infectious Diseases. 2016;16(34):1-5

7. Garg RK, Somvanshi DS. Spinal tuberculosis: A review. J Spinal Cord Med. 2011;34(5):440-454. doi:10.1179/2 045772311 Y.0000000023

8. Minhao Wu, MS, JiajiaSu, MS, et al. Skipped multifocal extensive spinal tuberculosis involving the whole spine. Medicine. 2018;97:3(e9692).

9. Osmanagic A, Emamifar A, Bang JC, et al. A rare case of pott's disease (spinal tuberculosis) mimicking metastatic disease in the southern region of Denmark. Am J Case Rep. 2016;17:384.

10. Thammaroj J, Kitkhuandee A, Sawanyawisuth K, et al. MR findings in spinal tuberculosis in an endemic country. J Med Imaging Radiat Oncol. 2014;58:267.

11. Khan F, Govender S. Sacroiliac Joint Involvement in Spinal Tuberculosis. SA Orthop J. 2018;17(3):47-52 